\title{
COMMENTARY
}

\section{Improving surgical outcomes: it is the destination not the journey}

\author{
Jonathan Wilson* and Simon Davies \\ See related research by Benes et al., http://ccforum.com/content/14/3/R118
}

\begin{abstract}
Studies have demonstrated that optimising the circulating volume reduces morbidity after major surgery. This optimisation is usually achieved through maximisation of the stroke volume guided by oesophageal Doppler. New monitoring parameters of preload responsiveness using information from the arterial trace are now showing some promise in achieving the same goal. The present commentary examines these new parameters with respect to improving outcomes for the high-risk surgical patient.
\end{abstract}

In the previous issue of Critical Care, Benes and colleagues have demonstrated improvements in outcomes for patients undergoing major intra-abdominal gastrointestinal or vascular surgery by giving additional fluid boluses in order to maintain a variation in stroke volume $(\mathrm{SVV})<10 \%$, rather than targeting the stroke volume itself [1].

Targeting elevated oxygen delivery or stroke volume improves outcome after high-risk surgery [2,3]. Protocols used in these studies have required either pulmonary artery catheterisation or the insertion of an oesophageal Doppler monitoring (ODM) probe. The pulmonary artery catheter is highly invasive, whilst the use of ODM is restricted to the anaesthetised or heavily sedated patient. Despite these limitations, ODM is considered a minimum standard of care for high-risk surgical patients [4].

In many respects, Benes group's findings echo those of ODM optimisation studies, in as much as the intervention group received $400 \mathrm{ml}$ more colloid than the control group, and the length of stay and complications were both reduced. What is different, however, is the monitoring parameter used as the targeted endpoint.

*Correspondence: jonathan.rjt.wilson@york.nhs.uk

Department of Anaesthesia, York Teaching Hospital, Wigginton Road, York YO31 8HE, UK
SVV is measured by pulse contour analysis (PCA), and is one of a group of parameters that estimate preload responsiveness - the other parameters being pulse pressure variation and systolic pressure variation. Benes and colleagues' study is now the third study showing that targeting preload responsiveness leads to improved outcomes after major surgery $[5,6]$.

We are all familiar with SVV whether we realise it or not. The swing on an arterial line trace in the operating theatre or in the intensive care unit alerts us to the possibility that a patient is hypovolaemic and may respond to a fluid bolus. SVV effectively automates this subjective observation. The effect of the increased intrathoracic pressure of mechanical ventilation is to decrease venous return to the right side of the heart, reducing right ventricular filling and output, leading to a reduced left ventricular stroke volume. This continuous challenge provided by mechanical ventilation allows estimation of the adequacy of preload, by monitoring changes in the stroke volume over a respiratory cycle, and the degree of variation seen in either the stroke volume or the pulse pressure indicates whether the subject would be responsive to fluid bolus by improving their stroke volume [7-9].

The benefits of a PCA system such as the Vigileo ${ }^{\mathrm{ma}}$, LiDCORapid $^{\mathrm{mm}}$ or $\mathrm{PiCCO}^{\mathrm{mm}}$ lie with the ease of use particularly in higher-risk patients, in whom invasive arterial monitoring is routine. In contrast to the ODM, a PCA technique can be used comfortably in awake patients allowing stroke volume optimisation in the postoperative environment, which has also shown to be of benefit [10]. These PCA systems are little affected by surgical movement or diathermy, and provide a simple, consistent, and defined end point as to whether fluid is required based on SVV being $>10 \%$ or $<10 \%$.

Preload responsiveness parameters are not without their limitations: they require constant tidal volumes of around 7 to $8 \mathrm{ml} / \mathrm{kg}$ and sinus rhythm; they are subject to problems with damping of the arterial trace; and up until now they had limited validation in terms of improved clinical outcome.

With the use of SVV in goal-directed therapy, Benes and colleagues have shown a significant reduction in 
postoperative morbidity [1], which in the control group was similar to other studies investigating high-risk patients. A relatively homogeneous study group has been investigated; and although single centre, the methodology is robust. This study has once again demonstrated that so-called standard haemodynamic parameters are not suitable targets to direct fluid therapy. It would be interesting to know the timing of fluid administration, as this has also been suggested as a factor in the improved outcomes [11]. Patients with dysrythmias, excluded from the benefits of preload responsiveness, are likely to be a true high-risk group, and it would be interesting to know whether stroke volume optimisation using PCA could be a useful alternative in this group. Studies are also required to determine optimal values of SVV or systolic pressure variation for predicting fluid responsiveness as determined by a significant rise in stroke volume measured by ODM, the current standard.

In summary, getting the preload right remains the cornerstone of goal-directed fluid therapy, and many of us believe it should be a minimum standard of care for the high-risk surgical patient. The range of tools to do the job is expanding to suit all tastes - so although the destination remains the same, it now seems the journey can be made on different paths.

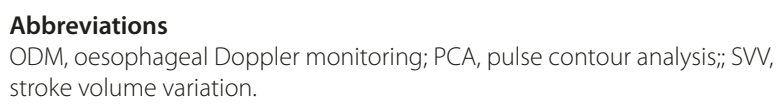

\section{Competing interests}

JW has received honoraria for lecturing from Deltex (manufacturers of an oesophageal Doppler device) and from LiDCO (manufacturers of a pulse contour analysis system). SD received sponsorship from LiDCO to help attend the 2009 International Anaesthetic Research Society Congress.
References

1. Benes J, Chytra I, Altmann P, Hluchy M, Kasal E, Svitak R, Pradl R, Stepan M: Intraoperative fluid optimization using stroke volume variation in high risk surgical patients: results of prospective randomized study. Crit Care 2010, 14:R118.

2. Poeze M, Greve JWM, Ramsay G: Meta-analysis of hemodynamic optimization: relationship to methodological quality. Crit Care 2005, 9:771-779.

3. Abbas SM, Hill AG: Systematic review of the literature for the use of oesophageal Doppler monitor for fluid replacement in major abdominal surgery. Anaesthesia 2008, 63:44-51.

4. Mowatt G, Houston G, Hernandez R, de Verteuil R, Fraser C, Cuthbertson B, Vale L: Systematic review of the clinical effectiveness and costeffectiveness of oesophageal Doppler monitoring in critically ill and highrisk surgical patients. Health Technol Assess 2008, 13:1-118.

5. Lopes MR, Oliveira, Pereira VOS, Lemos IPB, Auler JOC, Michard F: Goaldirected fluid management based on pulse pressure variation monitoring during high-risk surgery: a pilot randomized controlled trial. Crit Care 2007, 11:R100.

6. Mayer J, Boldt J, Mengistu AM, Rohm KD, Suttner S: Goal-directed intraoperative therapy based on autocalibrated arterial pressure waveform analysis reduces hospital stay in high-risk surgical patients: a randomized, controlled trial. Crit Care 2010, 14:R18.

7. Cannesson M, Musard H, Desebbe O, Boucau C, Simon R, Henaine R, Lehot J.: The ability of stroke volume variations obtained with Vigileo/FloTrac system to monitor fluid responsiveness in mechanically ventilated patients. Anesth Analg 2009, 108:513-517.

8. Biais M, Nouette-Gaulain K, Cottenceau V, Revel P, Sztark F: Uncalibrated pulse contour-derived stroke volume variation predicts fluid responsiveness in mechanically ventilated patients undergoing liver transplantation. Br J Anaesth 2008, 101:761-768.

9. Berkenstadt H, Margalit N, Hadani M, Friedman Z, Segal E, Villa Y, Perel A: Stroke volume variation as a predictor of fluid responsiveness in patients undergoing brain surgery. Anesth Analg 2001, 92:984-999.

10. Pearse R, Dawson D, Fawcett J, Rhodes A, Grounds, Bennett ED: Early goaldirected therapy after major surgery reduces complications and duration of hospital stay. A randomised, controlled trial. Crit Care 2005, 9:687-693.

11. Noblett SE, Snowden CP, Shenton BK, Horgan AF: Randomized clinical trial assessing the effect of Doppler-optimized fluid management on outcome after elective colorectal resection. Br J Surg 2006, 93:1069-1076.

doi:10.1186/cc9082

Cite this article as: Wilson J, Davies S: Improving surgical outcomes: it is the destination not the journey. Critical Care 2010, 14:177. 